УДК 621.311.26: 621.311 .68

\title{
ДВУХКОНТУРНЫЙ НАКОПИТЕЛЬ ЭНЕРГИИ ДЛЯ ГИБРИДНЫХ ЭНЕРГЕТИЧЕСКИХ СИСТЕМ С ВОЗОБНОВЛЯЕМЫМИ ИСТОЧНИКАМИ ЭНЕРГИИ
}

\author{
Обухов Сергей Геннадьевич1, \\ serob99@mail.ru
}
Плотников Игорь Александрович1, igorplt@tpu.ru

Ибрагим Ахмед ${ }^{1}$, ibragim@tpu.ru

\author{
Масолов Владимир Геннадьевич², \\ vdm-tech@mail.ru \\ 1 Национальный исследовательский Томский политехнический университет, \\ Россия, 634050, г. Томск, пр. Ленина, 30. \\ 2 ООО «ВДМ-техника», \\ Россия, 115093, г. Москва, ул. Павловская, 27, стр. 3, оф. 206.
}

\begin{abstract}
Актуальность. Актуальной задачей технико-экономического развития северных и восточных регионов России является обеспечение надежного и эффрективного электроснабжения потребителей, территориально расположенных в отдаленных, труднодоступных районах. Перспективным способом решения данной проблемы является применение гибридных энергетических систем с возобновляемыми источниками энергии. Характерной особенностью режимов гибридных систем, особенно $c$ высоким уровнем замещения топлива, является наличие пульсаций в зарядно-разрядных токах аккумуляторных батарей, используемых в качестве накопителей энергии. Эксплуатация аккумуляторных батарей в режиме импульсных токов приводит к быстрой деградации их характеристик и сокращению срока службы, что обуславливает снижение надежности системы электроснабжения и увеличение себестоимости генерируемой электроэнергии. Существенным недостатком гибридных систем, построенных по известным стандартным схемам, является неэфрфективное использование потенциала первичной возобновляемой энергии, что особенно критично для энергетических систем, территориально расположенных в районах с суровыми климатическими условиями. В статье предложены технические решения, позволяющие устранить обозначенные проблемы.

Цель: поиск и разработка технических решений, обеспечивающих повышение надежности и эфффективности гибридных энергетических систем с высоким уровнем замещения топлива.

Mетоды: математическое и компьютерное моделирование с использованием программной среды MatLab/Simulink.

Результаты. Предложен новый способ построения и алгоритм управления режимами гибридных энергетических систем, обеспечивающих повышение их надежности и энергетической эфффективности. Представлены результаты моделирования рабочих режимов гибридной электростанции с высоким уровнем замещения топлива, которые доказывают, что предложенный способ построения и алгоритм управления режимами обеспечивают надежное и эфффективное управление балансом мощности гибридной энергетической системы во всех возможных эксплуатационных режимах. Применение двухконтурного накопителя энергии с предложенным алгоритмом управления обеспечивает снижение интенсивности отказов гибридной энергетической системы на 5,7 \%, увеличение эксплуатационного ресурса аккумуляторных батарей на 50 \%, повышение эфффективности использования энергии возобновляемых источников на 28 \% в сравнении со стандартными способами построения гибридных электростанций.
\end{abstract}

\section{Ключевые слова:}

Гибридная энергетическая система, возобновляемье источники энергии, накопитель энергии, суперконденсатор, аккумуляторная батарея.

\section{Введение}

Приоритетным направлением развития современной энергетики является применение возобновляемых источников энергии (renewable energy sources - RES). Одной из наиболее перспективных технологий использования RES являются гибридные энергетические системы (hybrid renewable energy systems HRES), первоочередной областью практического применения которых являются децентрализованные системы электроснабжения потребителей, территориально расположенные в отдаленных, труднодоступных районах. Имеющийся опыт эксплуатации HRES показал, что их применение обеспечивает повышение надежности, экономической и экологической эффективности в сравнении с традиционными системами электроснабжения, реализуемыми на основе дизельгенераторных установок (diesel generator sets - DGS) [1-3].

В общем случае в составе HRES могут применяться самые разнообразные источники энергии, но наибольшее распространение получили системы с использованием фотоэлектрических (photovoltaic PV) и ветроэнергетических (wind turbine - WT) установок. Обусловлено это тем, что энергия Солнца и энергия ветрового потока повсеместно доступны. 
Энергетические установки PV и WT выпускаются на широкий диапазон мощностей и их можно максимально приблизить к месту конечного потребления энергии, что особенно важно для автономной энергетики.

Самыми сложными с точки зрения управления режимами, и в то же время наиболее эффективными по потреблению горюче-смазочных материалов, эксплуатационным затратам и экологической чистоте, являются энергетические системы с высоким уровнем замещения органического топлива [4]. Обязательным элементом таких энергосистем является накопитель энергии, применение которого позволяет значительно повысить эффективность электростанции за счет аккумулирования излишков энергии в периоды ее избытка и отдачи потребителю в периоды дефицита. Tребованиям HRES по объемам и времени хранения энергии наилучшим образом соответствуют аккумуляторные системы накопления энергии (battery energy storage systems - BESS), которые и получили наибольшее распространение в гибридных системах $[5,6]$.

Характерной особенностью рабочих режимов HRES, особенно с высоким уровнем замещения топлива, является изменение в широком диапазоне значений генерируемой и потребляемой мощности на различных временных интервалах, что обуславливает наличие пульсаций в зарядно-разрядных токах BESS, которые приводят к повышению их температуры, выкипанию электролита и коррозии электродов [7, 8]. Эксплуатация BESS в режиме импульсных токов приводит к быстрой деградации их характеристик и сокращению срока службы. В работе [9] представлены результаты экспериментальных исследований по определению срока службы свинцово-кислотной батареи гелевого типа в режимах заряда-разряда сглаженным и импульсным токами. В результате проведенных экспериментов установлено, что при эксплуатации батареи в импульсном режиме ее гарантированный срок службы сокращается практически в 2 раза. Аналогичные результаты получены в работе [10] для аккумуляторов, выпускаемых под торговой маркой Tesla. В работе [11] представлены результаты исследований причин отказов свинцово-кислотных, никель-кадмиевых и литий-ионных батарей, работающих в составе HRES. Авторами установлено, что одной из основных причин отказа батарей являются режимы заряда-разряда импульсными токами.

Высокая актуальность данной проблемы определяется тем, что именно BESS является самым «слабым» звеном HRES с точки зрения эксплуатационного ресурса. Гарантированный срок службы большей части основного энергетического оборудования HRES (WT, PV, DGS, силовые преобразователи), заявляемый их производителями, обычно составляет 20-25 лет. Срок службы BESS, как правило, не превышает 5-10 лет и только при условии их оптимальной эксплуатации. При этом финансовые затраты на BESS составляют существенную долю от общей стоимости энергетической системы. Например, в работе [12] установлено, что затраты на BESS составляют 52 \% от общих приведенных затрат на небольшую PV станцию для одного домохозяйства в Индонезии. В работе [13] приведены результаты исследований по оптимизации состава оборудования HRES, включающей WT и PV, предназначенной для электроснабжения объекта со средним энергопотреблением 5,6 кВт·ч/сутки. В результате исследований определено, что стоимость BESS составляет 38,58 \% от общей стоимости электростанции.

Цель настоящей работы состояла в поиске и разработке технических решений, обеспечивающих повышение надежности и эффективности HRES с высоким уровнем замещения топлива. В результате проведенных исследований предложен новый, оригинальный способ построения и алгоритм управления режимами HRES, обеспечивающие повышение еe надежности и энергетической эффективности.

\section{Сравнительный анализ схем построения, систем контроля и управления режимами гибридных энергетических систем}

С точки зрения базовой архитектуры возможны два основных подхода к построению HRES: с сопряжением генерирующих источников на постоянном и на переменном токе [14-16]. Однозначного ответа на вопрос «Какая архитектура лучше?» не существует, каждая из систем имеет свои достоинства и недостатки, и, соответственно, преимущественную область применения [17]. Решающее значение для достижения высоких уровней надежности и энергетической эффективности HRES имеет система контроля и управления $[18,19]$. Существует два основных варианта структурной организации систем управления HRES: централизованная и децентрализованная (распределенная) [15, 18, 19]. Важным достоинством HRES c распределенным управлением является высокая надежность, так как одноточечные неисправности не являются критичными. При этом ощутимо повышается универсальность системы, что позволяет относительно легко вносить изменения в ее конфигурацию и проводить замены неисправного оборудования.

Проведенный анализ известных топологий и систем управления HRES показал, что для энергетических систем с высоким уровнем замещения преимущественно применяются HRES с архитектурой на постоянном токе, распределенной системой управления и аккумуляторными системами хранения энергии $[15,19]$. Достоинствами данного технического решения являются: достаточно высокая надежность энергетической системы, высокое быстродействие, хороший уровень унификации. В то же время данное решение имеет и ряд недостатков, наиболее существенными из которых являются: необходимость применения преобразователей с согласованными техническими характеристиками, высокая сложность и стоимость преобразовательной техники, неэффективное использование потенциала первичной возобновляемой энергии. Последний недостаток особенно критичен для энергетических систем, территориально расположенных в районах с суровыми климатическими 
условиями. Обусловлен данный недостаток тем, что при стандартной схеме построения HRES управление балансом мощности в системе осуществляется за счет ограничения выходной электрической мощности установок RES и в системах с высоким уровнем замещения топлива безвозвратные потери энергии будут весьма существенными.

Имеющийся опыт эксплуатации HRES в районах с суровыми климатическими условиями свидетельствует о том, что большая часть генерируемой энергии расходуется на систему жизнеобеспечения электростанции: обогрев контейнера, технологического оборудования и т. п. [20]. В предлагаемой схеме построения HRES излишки энергии, генерируемой установками RES, рассеиваются на балластных сопротивлениях, что позволяет полезно ее использовать для различных хозяйственных нужд: нагрев воды, отопление и т. п.

\section{Описание предлагаемой архитектуры построения и способа управления режимами гибридных энергетических систем}

В результате проведенных исследований разработано новое техническое решение, обеспечивающее максимально эффективное использование потенциала RES и оптимизацию режимов заряда/разряда BESS. Идея предлагаемого решения состоит в применении пассивной системы стабилизации напряжения на сборной DC шине за счет подключения к ней суперконденсатора (supercapasitor - SC) и управления энергетическим балансом в системе с помощью комбинированного накопителя энергии (combined energy storage - CES), состоящего из двух идентичных аккумуляторных батарей (battery bank - BB), поочередно работающих в режиме заряда/разряда, и регулируемой балластной нагрузки (ballast load - BL) [21].

Предлагаемая обобщенная схема построения HRES представлена на рис. 1.

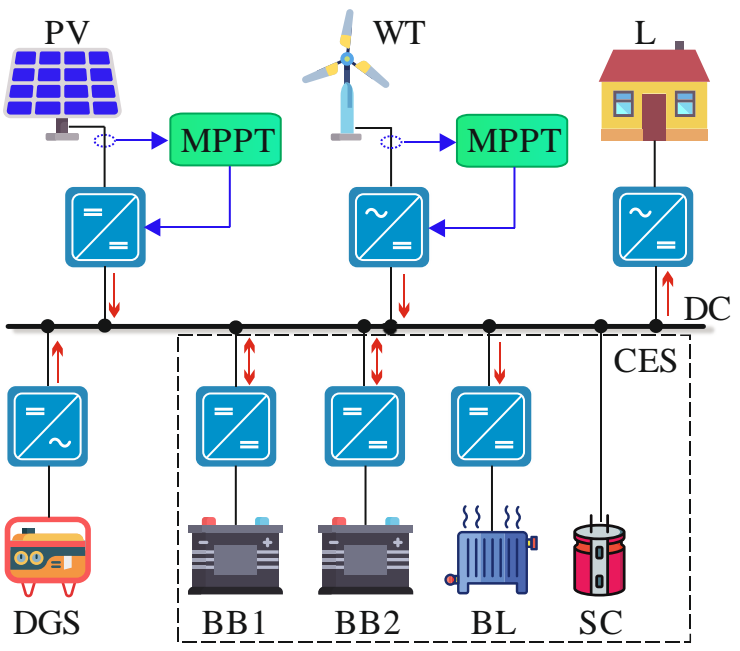

Рис. 1. Структурная схема гибридной электростанции с двухконтурным накопителем энергии

Fig. 1. Block diagram of a hybrid power plant with dualcircuit energy storage
Одной из основных компонент HRES при таком способе построения является DC-шина, функциональным назначением которой является сбор и распределении электрической энергии в замкнутой энергетической системе.

Уравнение баланса мощности для DC-шины запишется следующим образом:

$$
\begin{gathered}
P_{\mathrm{DC}}=V_{\mathrm{dc}} \cdot C \frac{V_{\mathrm{DC}}}{d t}= \\
\left(P_{\mathrm{DGS}}+P_{\mathrm{WT}}+P_{\mathrm{PV}}+P_{\mathrm{dch}}\right)-\left(P_{\mathrm{L}}+P_{\mathrm{BL}}+P_{\mathrm{ch}}\right)
\end{gathered}
$$

B общем случае баланс мощностей на шине определяется текущим соотношением генерируемой и потребляемой мощности. В качестве источников генерации могут использоваться: дизель-генераторные установки $\left(P_{\mathrm{DGS}}\right)$, ветроэнергетические $\left(P_{\mathrm{WT}}\right)$ и фотоэлектрические установки $\left(P_{\mathrm{PV}}\right)$, а также разрядная мощность ВВ $\left(P_{\mathrm{dch}}\right)$. Потребителями мощности являются: полезная нагрузка потребителя $\left(P_{\mathrm{L}}\right)$, балластная нагрузка $\left(P_{\mathrm{BL}}\right)$, зарядная мощность $\mathrm{BB}\left(P_{\mathrm{ch}}\right)$.

Неуправляемой переменной в уравнении (1) является величина мощности, потребляемой полезной нагрузкой $P_{\mathrm{L}}$. Для максимально эффективного использования доступной первичной энергии RES преобразователи WT и PV должны работать под управлением контроллеров поиска точки максимальной мощности (maximum power point tracking - MPРT) [22], соответственно величины генерируемой мощности $P_{\mathrm{WT}}$ и $P_{\mathrm{PV}}$ с точки зрения обеспечения энергетического баланса в системе также являются неуправляемыми переменными. Для управления балансом мощности и стабилизации величины напряжения DCшины предлагаемая стратегия управления предполагает использование двух основных управляемых источников мощности: дизель-генераторную установку $P_{\mathrm{DGS}}$ и аккумуляторную батарею, работающую в режиме разряда $P_{\mathrm{dch}}$. С учетом того, что в HRES с высоким уровнем замещения топлива возможны режимы, в которых величина генерируемой мощности установками RES может значительно превышать потребляемую мощность, в качестве дополнительного управляемого потребителя мощности в системе используется балластная нагрузка $P_{\mathrm{BL}}$. Вторая $\mathrm{BB}$, подключенная к шине в режиме отбора зарядной мощности может рассматриваться как частично управляемый потребитель мощности $P_{\mathrm{ch}}$.

На рис. 2 представлены упрощенные эквивалентные схемы замещения HRES для двух основных режимов стабилизации STAB1 и STAB2. В режиме стабилизации STAB1 (рис. 2, a) управление балансом мощности в системе обеспечивается разрядной $\mathrm{BB}$, в режиме стабилизации STAB2 (рис. 2, б) баланс мощности обеспечивается DGS. Так как управляемые источники мощности должны обеспечивать стабилизацию величины напряжения сборной DC-шины, они должны функционировать в режиме управляемых источников напряжения, все остальные энергетические установки представляются управляемыми и неуправляемыми источниками тока. 

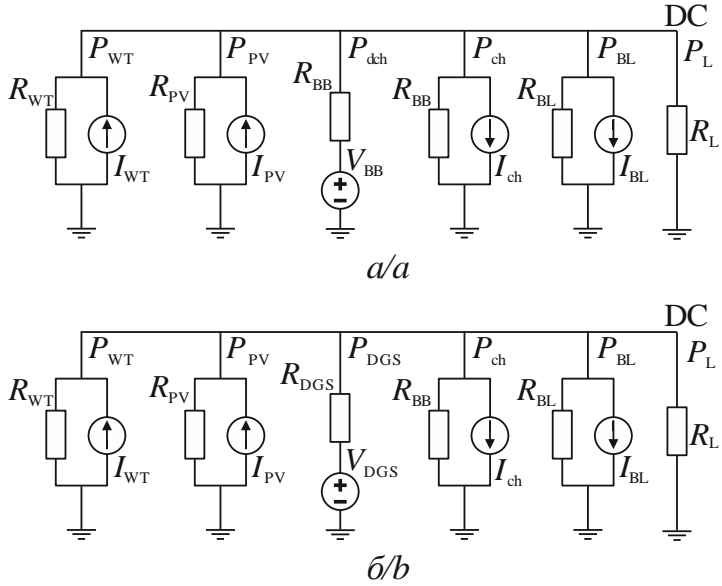

Pис. 2. Упрощенные эквивалентные схемы замещения гибридной энергетической системь

Fig. 2. Simplified equivalent circuits of substitution of the hybrid power system

B качестве критерия смены режимов работы HRES используется величина суммарной остаточной емкости BB $\sum \mathrm{SOC}$. Диаграмма перехода между режимами стабилизации схематично представлена на рис. 3 .

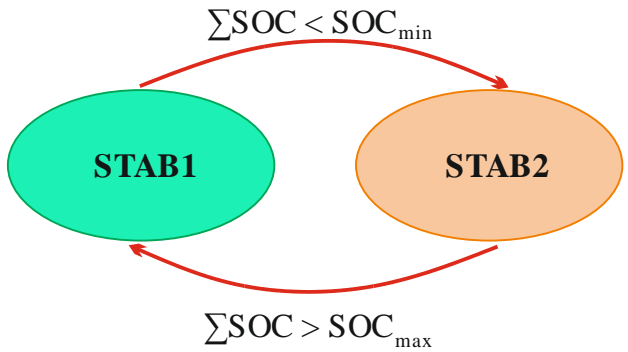

Рис. 3. Диаграмма перехода между режимами стабилизаичи

Fig. 3. Diagram of transition between the modes of stabilization

Предустановленные значения $\mathrm{SOC}_{\min }$ и $\mathrm{SOC}_{\max }$ определяются на этапе конфигурации системы и за-

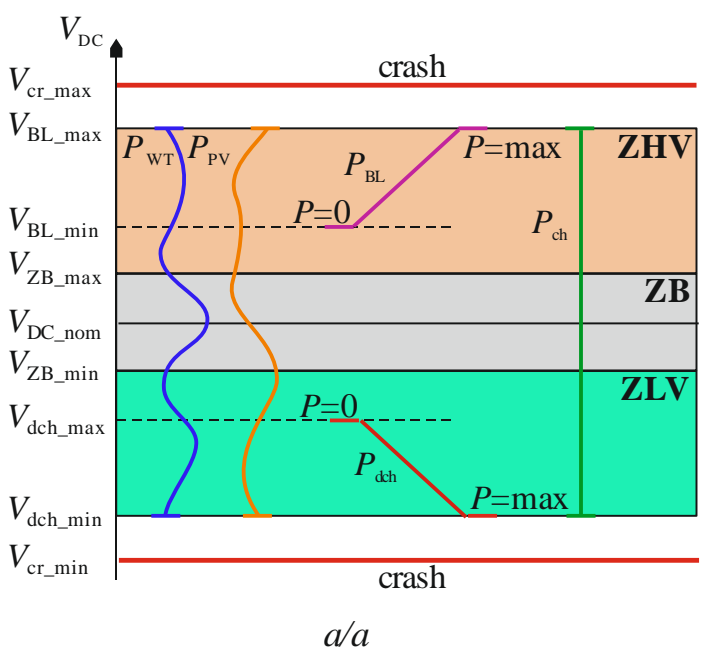

висят от типа используемых BESS, соотношения мощностей основных генерирующих источников, режимов эксплуатации электростанции, характера электрической нагрузки потребителя и т. д., и в общем случае подлежат оптимизации. В рассматриваемой HRES приняты следующие значения суммарной остаточной емкости обеих $\mathrm{BB}: \mathrm{SOC}_{\min }=125 \%$, $\mathrm{SOC}_{\max }=185 \%$ от их номинальной емкости. Выбор данных значений обусловлен тем, что в рассматриваемом проекте используются свинцово-кислотные BESS, что предопределяет необходимость ограничения их максимальной глубины разряда (depth of discharge - DOD) величиной DOD $=60-70 \%$ от номинальной емкости.

В базовом варианте используются три рабочих зоны напряжения DC-шины (рис. 4): буферная зона (buffer zone - ZB), зона высокого напряжения (high voltage zone - ZHV) и зона низкого напряжения (low voltage zone - ZLV). B ZHV суммарная мощность генерации установок RES превышает суммарную мощность потребления и в качестве стабилизирующего источника в режимах STAB1 и STAB2 используется регулируемая балластная нагрузка. Конструктивно балластная нагрузка представляет собой набор резисторов, которые подключаются к DC-шине через DC-DC преобразователь. Величина тока (соответственно мощности) преобразователя регулируется по следующему закону:

$$
I_{\mathrm{BL} \_ \text {ref }}=\frac{V_{\mathrm{DC}}-V_{\mathrm{BL} \_ \text {min }}}{V_{\mathrm{BL} \_ \text {max }}-V_{\mathrm{BL} \_ \text {min }}} \cdot I_{\mathrm{BL} \_ \text {max }} \cdot
$$

Уравнение (2) определяет линейное изменение потребляемой мощности балластной нагрузкой от 0 до номинального значения $P_{\mathrm{BL} \_ \text {max }}$ в диапазоне напряжений от $V_{\text {BL_min }}$ до $V_{\text {BL_max }}$. Для практической реализации данного закона управления требуется измерение двух электрических параметров режима: величины напряжения $\mathrm{DC}$-шины $V_{\mathrm{DC}}$ и входного тока преобразователя $I_{\mathrm{BL}}$.

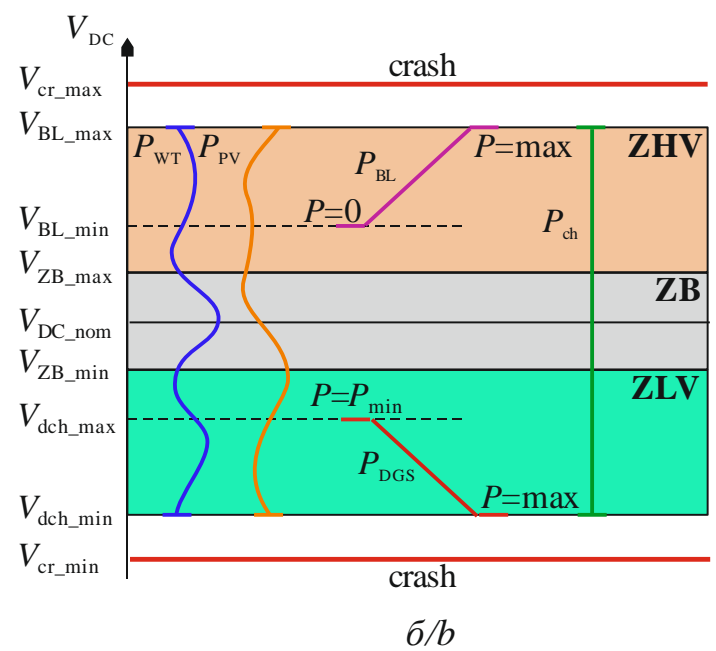

Pис. 4. Базовый вариант распределения рабочих зон напряжения в режиме стабилизащии: а) от аккумуляторной батареи; б) от дизель-генераторной установки

Fig. 4. Basic case of distribution of voltage zones in stabilization mode from: a) battery; b) diesel generator set 
Красными линиями на рис. 4 обозначена зона безаварийного функционирования электростанции. При выходе величины напряжения DC-шины за пределы заданных напряжений $V_{\text {cr_min }}$ и $V_{\text {cr_max }}$ режим работы энергетической системы инициируется как авария (crash), которая ликвидируется средствами противоаварийной автоматики.

B ZLV потребляемая мощность превышает суммарную мощность генерации установок RES и стабилизирующим источником в режиме STAB1 является $\mathrm{BB}$, работающая в режиме разряда на DC-шину (рис. $4, a$ ). Величина тока (соответственно мощности) преобразователя разрядной ВВ в этом режиме регулируется по следующему закону:

$$
I_{\text {dch_ref }}=\frac{V_{\text {dch_max }}-V_{\text {DC }}}{V_{\text {dch_max }}-V_{\text {dch_min }}} \cdot I_{\text {dch_max }} .
$$

Уравнение (3) определяет линейное изменение генерируемой мощности разрядной $\mathrm{BB}$ от 0 до номинального значения $P_{\text {dch_max }}$ в диапазоне напряжений от $V_{\text {dch_max }}$ до $V_{\text {dch_min. }}$ В качестве входных сигналов управления используется величина напряжения DCшины $V_{\mathrm{DC}}$ и значение выходного тока преобразователя $I_{\text {dch. }}$.

$\mathrm{ZB}$ используется для надежного разделения режимов работы регулирующих источников в системе, кроме того ее применение позволяет минимизировать (в идеале исключить) количество переходов из ZHV в $Z L V$, которые могут привести к неустойчивому или колебательному режиму работы системы управления.

B режиме STAB2 (рис. 4, б) основным регулирующим источником энергосистемы является DGS, для сохранения эксплуатационного ресурса которой необходимо минимизировать число ее включений и отключений. Кроме того, в рабочем режиме DGS необходимо обеспечить ее загрузку на уровне не ниже $25 \%$ от ее номинальной мощности. В базовом варианте распределения рабочих зон напряжения для режима STAB2 принят к рассмотрению вариант гарантированного заряда одной $\mathrm{BB}$, а вторая ВВ переводится в режим заряда только после полного заряда первой.

В соответствии с принятым алгоритмом, величина тока (соответственно мощности) преобразователя DGS регулируется по следующему закону:

$$
\begin{gathered}
I_{\text {DGS_ref }}=\frac{V_{\text {dch_max }}-V_{\text {DC }}}{V_{\text {dch_max }}-V_{\text {dch_min }}} \times \\
\times\left[I_{\text {DGS_max }}-I_{\text {DGS_min }}\right]+I_{\text {DGS_min }} .
\end{gathered}
$$

Практическая реализация условия (4) кроме гарантированного заряда одной ВВ за время включения DGS обеспечивает и приемлемый коэффициент загрузки дизельного двигателя. Это достигается ограничением и удержанием тока DGS не ниже некоторого предустановленного значения $I_{\text {DGS_min, }}$ соответствующего минимально допустимой загрузке дизеля, например $25 \%$ от номинальной мощности. Технически данное требование реализуется сужением диапа- зона регулирования выходного преобразователя DGS за счет ограничения значений коэффициента заполнения.

Необходимо отметить, что для реализации предложенных алгоритмов управления необходимо выполнение ряда важных соотношений, обеспечивающих эксплуатацию энергетической системы в пределах безопасных рабочих зон.

1. Величина максимально допустимой разрядной мощ-

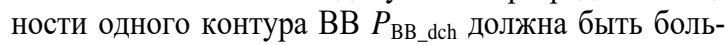
ше максимальной электрической нагрузки $P_{\mathrm{L} \_ \text {max }}$ :

$$
P_{\mathrm{BB} \_ \text {dch }} \geq P_{\mathrm{L}_{-} \max } ;
$$

2. Номинальная (максимальная) величина балластной нагрузки должна удовлетворять следующему уравнению:

$$
P_{\mathrm{BL} \_ \text {max }} \geq P_{\mathrm{WT}}+P_{\mathrm{PV}}-P_{\mathrm{L}_{-} \text {min }} \text {. }
$$

Частным случаем данного уравнения является условие равенства мощности минимальной электрической нагрузки нулю $P_{\mathrm{L}_{-} \min }=0$.

3. Номинальная мощность DGS должна обеспечивать покрытие максимальной электрической нагрузки и необходимую зарядную мощность ВВ:

$$
P_{\text {DGS_nom }} \geq P_{\mathrm{L}_{-} \max }+P_{\text {ch }} \text {. }
$$

Идея предлагаемой логики управления режимами HRES состоит в переключении BВ с режима заряда в режим разряда и обратно по заданным пороговым значениям ее остаточной емкости. Соответственно, для практической реализации данной логики управления необходим постоянный контроль величины остаточной емкости каждой ВВ в режиме реального времени. Кроме того, необходимо обеспечить контроль и суммарной остаточной емкости $\mathrm{BB} \sum \mathrm{SOC}$, величина которой является критерием для смены режимов работы энергетической системы (рис. 3).

Логику предлагаемого способа управления режимами HRES поясняет рис. 5, на котором представлены блок-схема алгоритма управления режимами (рис. $5, a$ ) и силовые схемы подключения BESS и DGS к сборной DC-шине (рис. 5, б).

Одним из возможных вариантов схемотехнического построения контроллера CES является разделение функций локального и стратегического управления преобразователями. По аналогичной схеме может быть построена и система управления преобразователем DGS. При применении данного подхода сигналы непосредственного управления ключами преобразователей $\left(I_{\text {ch_ref }}, I_{\text {dch_ref }}, I_{\text {DGS_ref }}\right)$ формируются по локальной токовой петле в зависимости от текущих значений напряжения DC-шины и выходного (входного) тока соответствующего преобразователя, а сигналы управления, обеспечивающие переключение режимов работы (Gate_BB1, Gate_BB2, DGS_on/off), формируются логическим блоком контроллера в зависимости от текущих значений остаточной емкости ВB.

На силовой схеме подключения стабилизирующих источников (рис. 5, б) для большей наглядности показано, что сигналы управления, формируемые логиче- 
ским блоком контроллера, обеспечивают непосредственное включение/отключение преобразователей BB и DGS за счет применения дополнительных ключей. В реальных схемах построения преобразователей применение дополнительных ключей не является обязательным, так как процессы включения/отключения преобразователей могут быть легко обеспечены за счет управления транзисторами их силовой части.
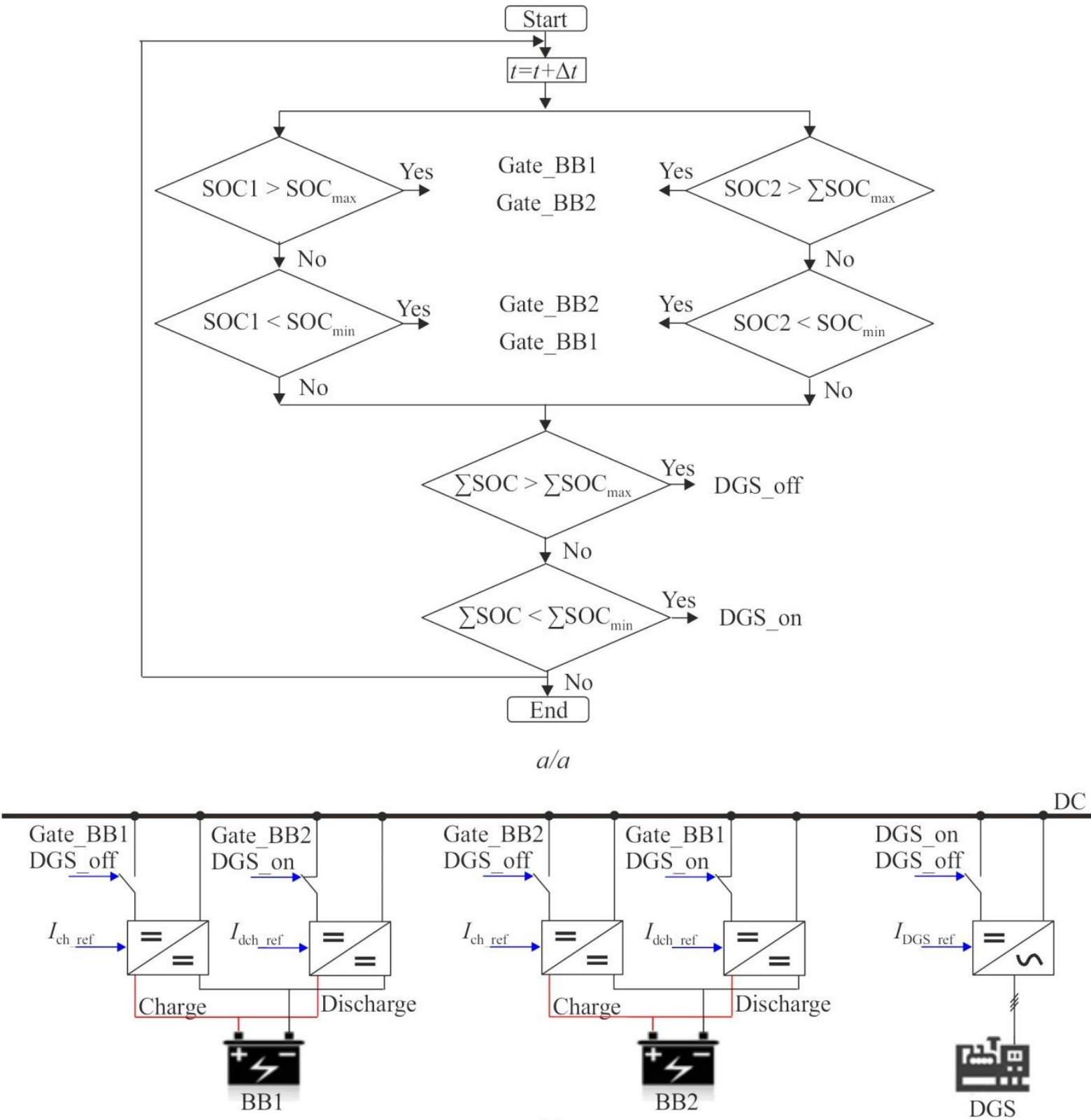

$\sigma / b$

Рис. 5. Логика предлагаемого способа управления режимами гибридной энергетической системь: а) блок-схема алгоритма управления; б) силовые схемы подключения стабилизирующих источников

Fig. 5. Logic of the proposed method of controlling the modes of the hybrid energy system: a) flow chart of the control algorithm; $b$ ) power circuits of the stabilizing sources

\section{Методы и объект исследования}

В качестве основного инструмента исследований в данной работе использовался программный комплекс MATLAB/Simulink, в среде которого в соответствии со структурной схемой HRES (рис. 1) были разработаны и реализованы математические модели всех основных компонентов рассматриваемой технической системы. В составе комплексной модели HRES можно выделить пять типов моделей: модели первичных энергоносителей (модель ветрового потока и солнечного излучения), модели энергетических установок (DGS, WT, PV), модели аккумулирующих устройств (SC, BESS), модели потребителей энергии (L, BL), модели силовых полупроводниковых преобразователей. 
В данной работе использовались динамические модели компонентов, построенные на основе уравнений, описывающих физические процессы преобразования энергии. Подробное описание моделей компонентов HRES, которые использовались при проведении исследований, приведено в работах [23-27]. Модели компонентов выполнены в виде отдельных функциональных блоков, что обеспечивает возможность построения и исследования режимов работы HRES произвольной конфигурации [28].

В качестве объекта исследования в настоящей работе принята автономная HRES, территориально расположенная в районе г. Томска, состоящая из WT, номинальной мощностью $10 \mathrm{\kappa Bт}\left(V_{\min }=3 \mathrm{~m} / \mathrm{c}, V_{\text {nom }}=9 \mathrm{~m} / \mathrm{c}\right)$, $\mathrm{PV}$ на базе солнечной батареи из 18 фотоэлектрических модулей Sunways FSM 340M, дизельгенераторного агрегата Geko 20012 ED-S/DEDA, номинальной мощностью 16 кBт, CES на базе аккумуляторов MONBAT 12MVR200 в количестве 20 штук на один контур и суперконденсаторного модуля из трех последовательно соединенных суперконденсаторов МСК-8-112, общей емкостью 2,7 Ф.

Для моделирования электрической нагрузки HRES использовался характерный суточный график нагрузок коммунально-бытового характера с максимумом в 10 кВт, величина максимальной балластной нагрузки принята равной 16,2 кВт. Рассматривался вариант PV c жестко зафиксированной солнечной батареей, ориентированной на Юг и установленной под углом $56,5^{\circ}$ к горизонту.

\section{Результаты и их обсуждение}

Для проверки работоспособности и апробации предложенных технических решений использовались
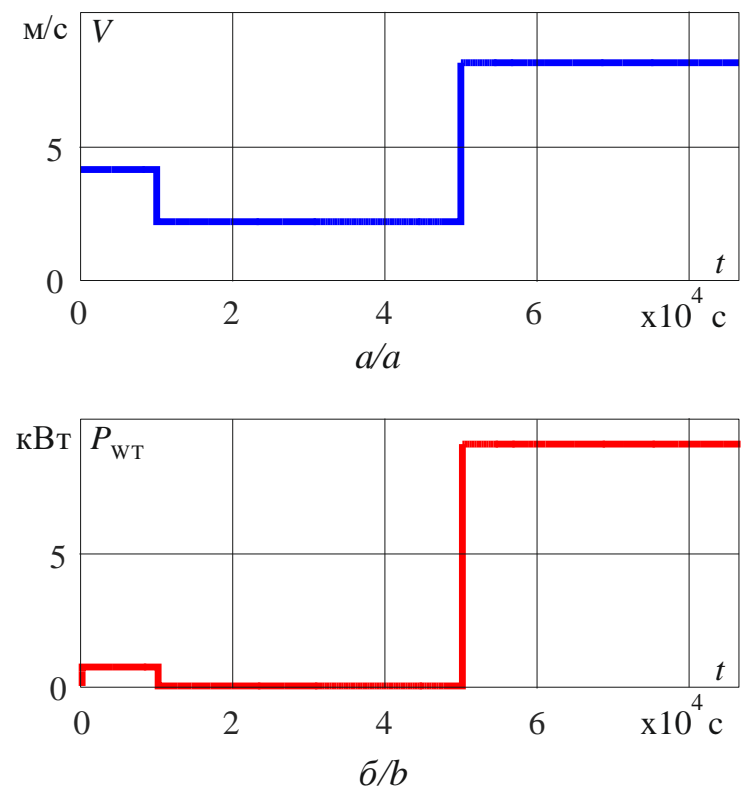

результаты компьютерного моделирования рабочих режимов HRES. Был разработан план исследований, в соответствии с которым проведена серия вычислительных экспериментов, моделирующих статические и динамические режимы HRES, возможные при ее эксплуатации.

В качестве примера на рис. 6 показаны результаты моделирования выходной электрической мощности установок RES ( $\mathrm{P}_{\mathrm{PV}}$ и $P_{\mathrm{WT}}$ ) на суточном временном интервале $(86400 \mathrm{c})$. Суточные изменения солнечной радиации $(G)$ соответствуют дню зимнего солнцестояния со средними условиями облачности. Скорость ветра $(V)$ задана ступенчатой функцией с амплитудой от 2 до $8 \mathrm{~m} / \mathrm{c}$, изменяющейся в моменты модельного времени 10000 и $50000 \mathrm{c}$.

Явное задание скорости ветра, остающейся неизменным на длительных интервалах времени, позволяет решить две задачи: с одной стороны значительно сократить требования к вычислительным ресурсам компьютера и тем самым уменьшить время моделирования; с другой стороны позволяет выделить для последующего анализа требуемые режимы функционирования CES и всей энергетической системы.

Результаты моделирования рабочих режимов HRES для заданных характеристик первичных энергоносителей представлены на рис. 7. На рисунке показаны графики изменения разрядного тока $\left(I_{\mathrm{dch}}\right)$, тока заряда $\left(I_{\mathrm{ch}}\right)$ и остаточной емкости (state of charge $\mathrm{SOC)}$ аккумуляторных батарей BB1 и BB2, напряжения сборной шины $\left(V_{\mathrm{DC}}\right)$, втекающих и вытекающих токов DC-шины: суммарного тока от установок RES $\left(I_{\mathrm{RES}}\right)$, тока полезной $\left(I_{\mathrm{L}}\right)$ и балластной $\left(I_{\mathrm{BL}}\right)$ нагрузок, тока DGS (I $\left.I_{\mathrm{DGS}}\right)$.
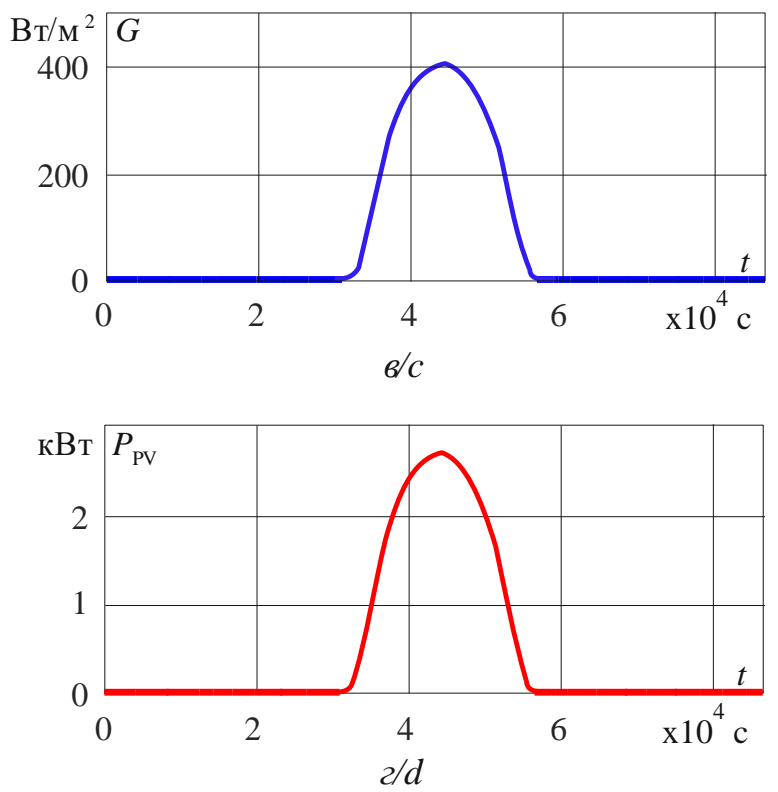

Рис. 6. Результаты моделирования выходной электрической мощуности установок возобновляемой энергетики на суточном временном интервале: а) скорость ветра; б) мощность ветроэнергетической установки; в) солнечная радиаџия; г) мощность солнечной батареи

Fig. 6. Simulation results of the output power of the renewable energy installations on the daily time interval: a) wind speed; b) wind power capacity; c) solar irradiance; d) solar power 

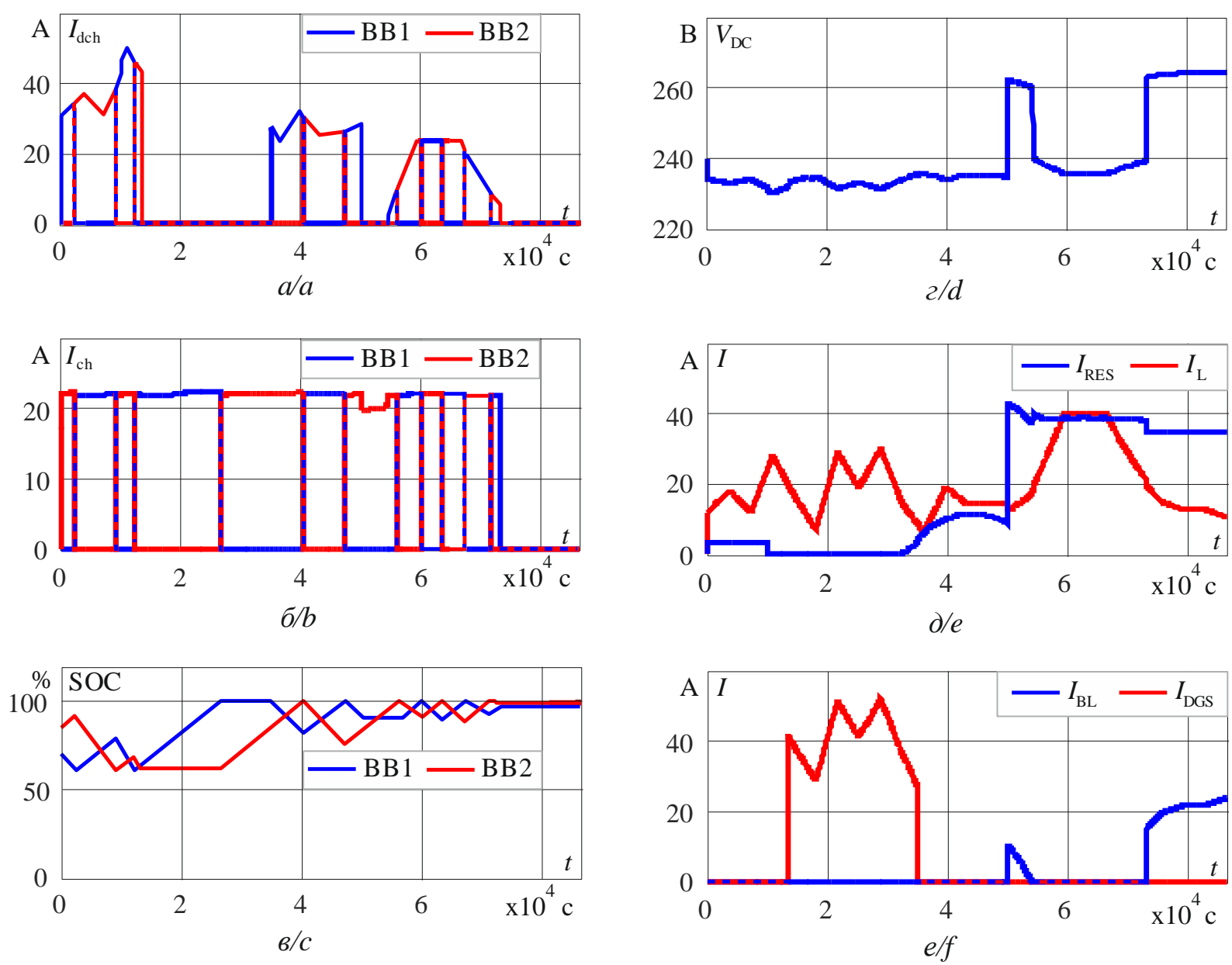

Рис. 7. Результаты моделирования рабочих режимов гибридной энергетической системы на суточном временном интервале: а) разрядный ток аккумуляторных батарей; б) зарядный ток аккумуляторных батарей; в) остаточная емкость аккумуляторных батарей; г) напряжение сборной шины; д) суммарный ток установок возобновляемой энергетики и ток нагрузки; е) ток балластной нагрузки и ток дизель-генераторной установки

Fig. 7. Results of simulation of operating modes of a hybrid energy system on the daily time interval: a) battery discharge current; b) battery charging current; c) residual battery capacity; d) busbar voltage; e) total current of renewable energy installations and load current; f) ballast current and diesel generator set current

Анализ полученных графиков (рис. 7) показывает, что на временном интервале от 0 до 50000 с мощности, генерируемой установками RES, недостаточно для полного покрытия электрической нагрузки. Это приводит к тому, что в момент времени около 14000 с обе ВВ оказываются разряженными ниже допустимого порогового уровня ( $\left.\sum \mathrm{SOC}<125 \%\right)$. Логический блок контроллера формирует управляющий сигнал на запуск DGS, и на интервале времени от 14000 до 35000 с управление балансом мощности в энергетической системе обеспечивается DGS. Аккумуляторные батареи BB1 и BB2 на этом временном интервале поочередно заряжаются, что хорошо видно из представленных на рис. 7 графиков их остаточной емкости SOC. При достижении суммарного заряда ВВ заданного порогового уровня ( $\left.\sum \mathrm{SOC}>185 \%\right)$ DGS отключается и дальше регулирование баланса мощности обеспечивается разрядной ВВ.

На интервалах модельного времени от 50000 до 54000 с и от 73000 с до конца рассматриваемых суток суммарная мощность, генерируемая установками RES, превышает мощность, потребляемую полезной нагруз- кой и заряжаемой ВВ. В соответствии с заданным алгоритмом (рис. 4) стабилизация энергетического баланса в системе в данных режимах обеспечивается регулированием мощности балластной нагрузки.

Анализ результатов моделирования свидетельствует о том, что предложенный алгоритм обеспечивает устойчивую работу энергетической системы в режимах STAB1 и STAB2, величина напряжения DCшины не выходит за пределы заданных рабочих зон, все преобразователи работают в штатном режиме.

В качестве наиболее сложных тестов проверки работоспособности алгоритма управления CES использовались результаты моделирования суточного рабочего режима HRES с учетом турбулентной составляющей скорости ветра. Турбулентная составляющая ветрового потока приводит к появлению широкополосной пульсации выходной мощности WT, что может способствовать потере динамической устойчивости энергетической системы. Однако такие эксплуатационные режимы являются типичными для HRES, соответственно их анализ является обязательным. 

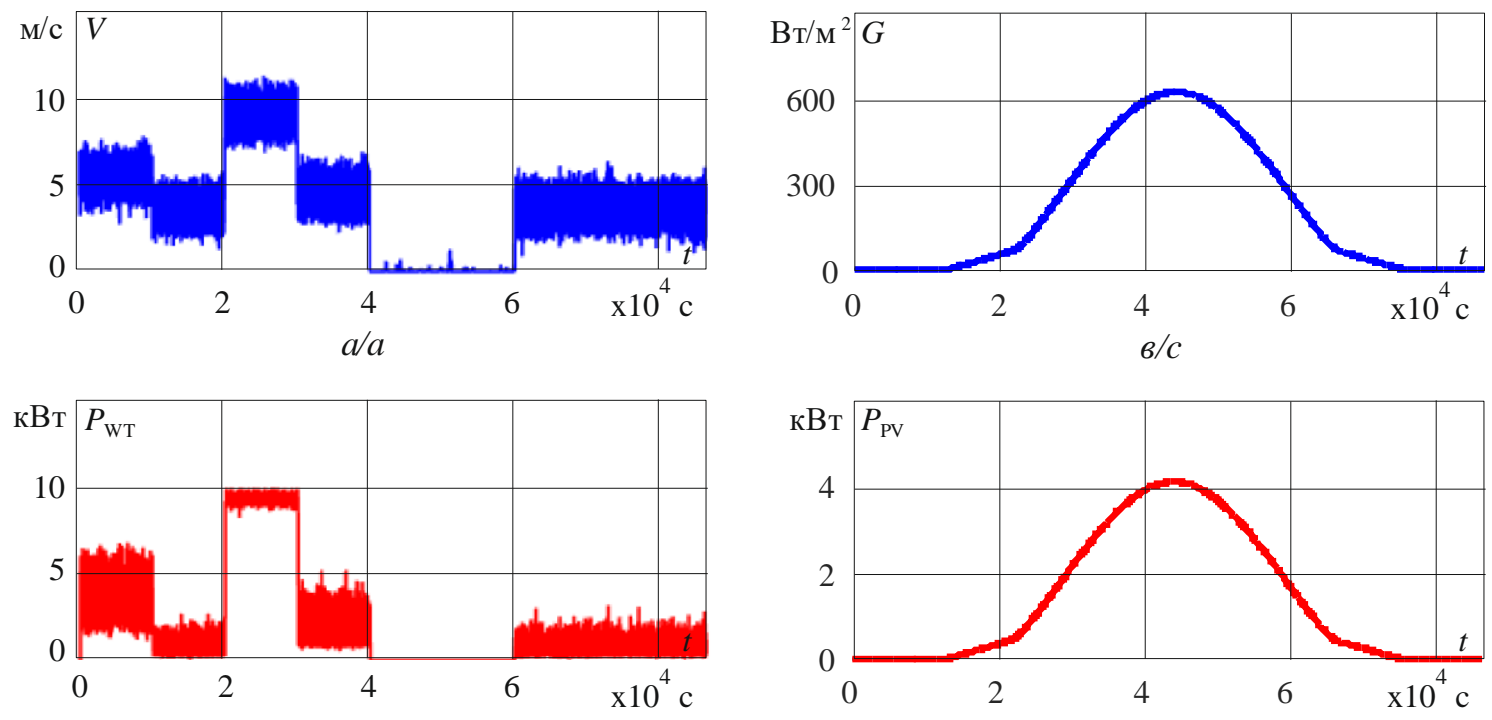

$6 / b$

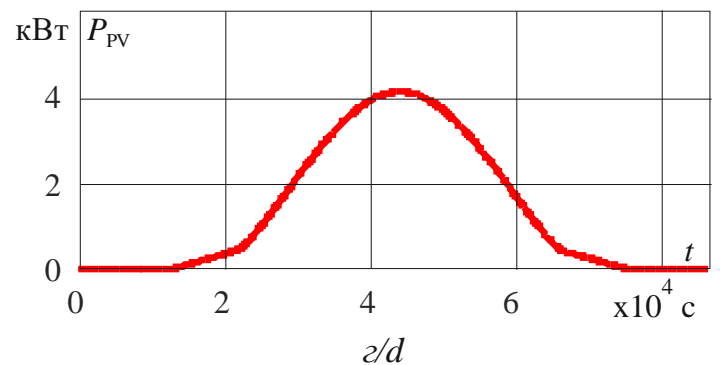

Рис. 8. Результаты моделирования выходной электрической мощности установок возобновляемой энергетики на суточном временном интервале: а) скорость ветра; б) мошность ветроэнергетической установки; в) солнечная радиация; г) мочиноть солнечной батареи

Fig. 8. Simulation results of the output power of the renewable energy installations on the daily time interval: a) wind speed; b) wind power capacity; c) solar irradiance; d) solar power
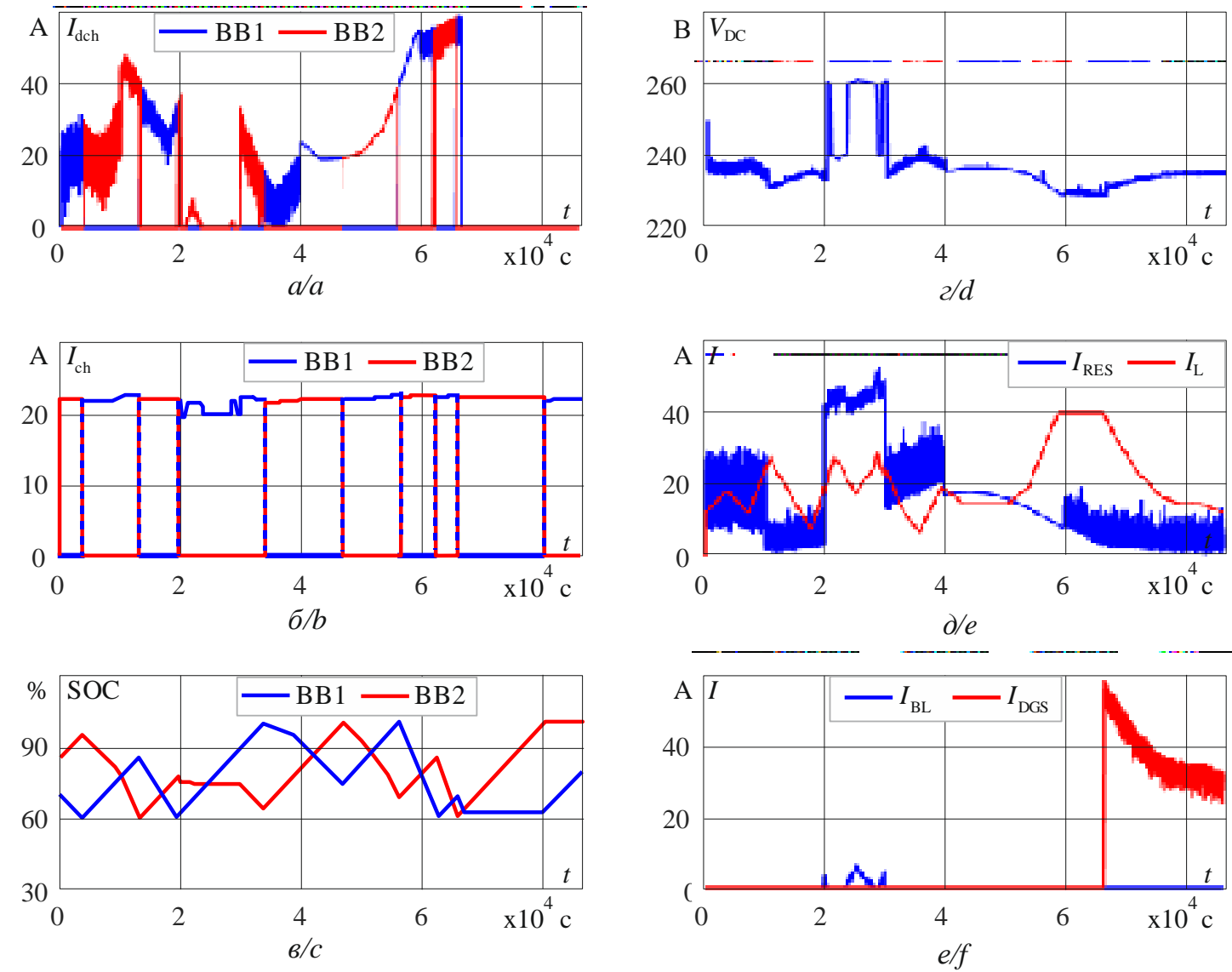

Рис. 9. Результаты моделирования рабочих режимов гибридной энергетической системы на суточном временном интервале: а) разрядный ток аккумуляторных батарей; б) зарядный ток аккумуляторных батарей; в) остаточная емкость аккумуляторных батарей; г) напряжение сборной шины; д) суммарный ток установок возобновляемой энергетики и ток нагрузки; е) ток балластной нагрузки и ток дизель-генераторной установки

Fig. 9. Simulation results of operating modes of a hybrid energy system on the daily time interval: a) battery discharge current; b) battery charging current; c) residual battery capacity; d) busbar voltage; e) total current of renewable energy installations and load current; f) ballast current and diesel generator set current 
На рис. 8, 9 представлены результаты моделирования суточного режима работы HRES с учетом турбулентной составляющей скорости ветра.

В данном вычислительном эксперименте рассматривается день летнего солнцестояния со средними условиями облачности, средняя скорость ветра в течение суток скачкообразно изменяется от 0 до $10 \mathrm{~m} / \mathrm{c}$ (рис. 8), что соответствует всему рабочему диапазону моделируемой WT. Как видно из представленных на рис. 9 графиков, система управления CES обеспечивает надежное и эффективное управление балансом мощности в изолированной энергетической системе во всех возможных эксплуатационных режимах.

Результаты проведенных вычислительных экспериментов подтвердили работоспособность предложенной архитектуры и алгоритма управления режимами автономной HRES с высоким уровнем замещения топлива.

Достоинствами рассмотренного алгоритма управления CES является его относительная простота и универсальность. Данный алгоритм обеспечивает режимы заряда ВВ стабильным током и минимизирует режимы переключения ВВ из режимов заряд в режим разряд и обратно, чем обеспечивается их сохранность и максимальный эксплуатационный ресурс.

Предложенная архитектура построения HRES позволяет использовать в ее составе силовые полупроводниковые преобразователи с несогласованными характеристиками и простой схемотехникой. С точки зрения эксплуатационной надежности HRES является достаточно живучей, так как никакой информационной связи между преобразователями установок RES не требуется, отказ любого преобразователя или генерирующего источника, кроме выходного инвертора, не нарушает общей работоспособности энергетической системы. Обеспечивается простота изменения конфигурации системы путем добавления/исключения в состав электростанции генерирующих установок разного типа и разных производителей без необходимости изменения настроек системы управления.

Выполненный расчет надежности HRES показал, что применение CES с предложенным алгоритмом управления режимами обеспечивает снижение интенсивности отказов энергетической системы на $5,7 \%$ и увеличение эксплуатационного ресурса ВB на 50 \%. Для расчета показателей надежности HRES применялся логиковероятностный метод на основе структурных схем надежности [29], в качестве исходных данных использовались типичные показатели надежности элементов исследуемой системы, установленные по технической

\section{СПИСОК ЛИТЕРАТУРЫ}

1. Renewables 2018 Global Status Rep ort. Renewable Energy Policy Network for the 21st Century. - 2018. - 325 p. URL http://www.ren21.net/ (дата обращения: 29.04.2019).

2. Sawle Y., Gupta S.C., Bohre A.K. Review of hybrid renewable energy systems with comparative analysis of off-grid hybrid system // Renewable and Sustainable Energy Reviews. - 2018. V. 81 (2). - P. 2217-2235.

3. Архипова О.В., Ковалев В.З., Хамитов Р.Н. Методика моделирования регионально обособленного электротехнического комплекса // Известия Томского политехнического университета. Инжиниринг георесурсов. - 2019. - Т. 330. - № 1. C. $173-180$. спецификации [30], эксплуатационный ресурс ВВ определен по результатам исследований [9-11].

Анализ годового энергетического баланса рассматриваемой HRES, территориально расположенной в районе г. Томска, показал, что применение CES с предлагаемым алгоритмом управления обеспечивает увеличение выработки энергии установками RES, а соответственно повышение эффективности использования энергии на $28 \%$ в сравнении со стандартными способами построения HRES. Следует отметить, что значение данного показателя во многом зависит от энергетического потенциала RES в месте расположения электростанции, соотношения установленных мощностей и технических характеристик основного энергетического оборудования и нагрузки, и может достигать значений до $60 \%$ [31].

В настоящее время на производственной площадке ООО «ВДМ-техника» изготовлен экспериментальный образец CES на номинальную мощность 15 кВт на основе аккумуляторных и суперконденсаторных модулей, разработан комплект эскизной конструкторской документации, программа и методика его исследовательских испытаний. В 2020 г. запланировано проведение испытаний экспериментального образца CES в составе стенда-полигона локальной системы электроснабжения с возобновляемыми источниками энергии, что позволит оценить возможность и эффективность его практического применения в реальных энергетических системах, проверить принятые компоновочные решения, а также найти технические решения, обеспечивающие улучшение его конструкции.

\section{Заключение}

Результаты проведенных исследований показали, что предложенный способ построения и алгоритм управления режимами HRES обеспечивают надежное и эффективное управление балансом мощности в HRES во всех возможных эксплуатационных режимaх. Достоинствами предложенных технических решений являются: обеспечение режимов заряда аккумуляторных батарей стабильным током, минимизация режимов неполного заряда/разряда и эффективное использование потенциала первичной возобновляемой энергии.

Работа выполнена при финансовой поддержке Министерства науки и высшего образования Российской Федерации. Уникальный идентификатор работ RFMEFI57617Х0098 (Соглашение о предоставлении субсидии от 26.09.2017 г. № 075-11-2018-181, внутренний номер соглашения 14.576.21.0098).

4. Salas V., Suponthana W., Salas R.A. Overview of the off-grid photovoltaic diesel batteries systems with AC loads // Applied Energy. - 2015. - V. 157 (1). - P. 195-216.

5. Battery storage for renewables: market status and technology outlook // International Renewable Energy Agency. URL: https://www.irena.org/-

/media/Files/IRENA/Agency/Publication/2015/IRENA_Battery_ Storage_report_2015.pdf (дата обращения: 29.04.2019).

6. Eller A., Gauntlett D. Energy storage trends and opportunities in emerging markets. URL: https://www.esmap.org/sites/default/ files/esmap-files/7151-IFC-EnergyStorage-report.pdf (дата обращения: 29.04.2019)

7. Hu X., Martinez C.M., Yang Y. Charging, power management, and battery degradation mitigation in plug-in hybrid electric 
vehicles: a unified cost-optimal approach // Mechanical Systems and Signal Processing. - 2017. - V. 87 (8). - P. 4-16.

8. Kan S.Y., Verwaal M., Broekhuizen H. The use of batterycapacitor combinations in photovoltaic powered products // Journal of Power Sources. - 2006. - V. 162 (2). - P. 971-974.

9. Optimal hybridization and amortized cost study of battery/supercapacitors system under pulsed loads / A. Lahyania, A. Sari, I. Lahbib, P. Venet // Journal of Energy Storage. 2016. - V. 6. - P. 222-231.

10. Hund T. Capacity loss in PV batteries and recovery procedures // Photovoltaic System Applications Department, Sandia National Laboratories. - 12 p. URL: https://pdfs.semanticscholar.org/87fd /d2343a6665f1251ee0c645fd3442f2e340e1.pdf?_ga=2.91045971 $.85236334 .1578964892-1940863712.1578964892$ (дата обращения: 29.04.2019)

11. Jossen A., Garche J., Sauer D.U. Operating conditions of batteries in PV applications // Solar Energy. - 2004. - V. 76. P. 759-769.

12. Life cycle cost analysis of a standalone PV system / A.Q. Jakhrani, A-K. Othman, A.R.H. Rigit, S.R. Samo // IEEE 2012 Int. Conf. Green Ubiquitous Technol. - Jakarta, Indonesia, 2012. - P. 82-85.

13. Optimum design of an off-grid hybrid renewable energy system for an office building / A. Ataei, M. Nedaei, R. Rashidi, C. Yoo // Journal of Renewable and Sustainable Energy. - 2015. V. 7 (053123). -27 p. DOI: 10.1063/1.4934659.

14. A review of hybrid renewable/alternative energy systems for electric power generation: configurations, control, and applications M.H. Nehrir, C. Wang, K. Strunz, H. Aki, R. Ramakumar, J. Bing, Z. Miao, Z. Salameh // IEEE Transactions on Sustainable Energy. - 2011. - V. 2 (4). - P. 392-403.

15. Upadhyay S., Sharma M.P. A review on configurations, control and sizing methodologies of hybrid energy systems // Renewable and Sustainable Energy Reviews. - 2014. - V. 38. - P. 47-63.

16. Sawle Y., Gupta S.C., Bohre A.K. PV-wind hybrid system: a review with case study // Cogent Engineering. - 2016. - V. 3. P. 1-21.

17. Обухов С.Г., Плотников И.А. Сравнительный анализ схем автономных электростанций, использующих установки возобновляемой энергетики // Промышленная энергетика. 2012. - № 7. - C. 46-51.

18. Energy management strategies in hybrid renewable energy systems: a review / L. Olatomiwa, S. Mekhilef, M.S. Ismail M. Moghavvemi // Renewable and Sustainable Energy Reviews. - 2016. - V. 62. - P. 821-835.

19. Chauhan A., Saini R.P. A review on integrated renewable energy system based power generation for stand-alone applications: configurations, storage options, sizing methodologies and control // Renewable and Sustainable Energy Reviews. - 2014. - V. 38. P. $99-120$.

20. A hybrid power plant based on renewables and electrochemical energy storage and generation systems for decentralized electricity supply of the northern territories / A.S. Grigoriev, V.V. Skorlygin, S.A. Grigoriev, D.A. Melnik, M.N. Filimonov // International Journal of Electrochemical Science. - 2018. - V. 13. - P. 1822-1830.
21. Система автономного электроснабжения: пат. Рос. Федерация № 2638025; заявл. 10.01.2017; опубл. 11.12.2017, Бюл. № $35 .-5 \mathrm{c}$.

22. Подход к построению адаптивного алгоритма экстремального регулирования мощности в системе солнечной энергетики / С.Г. Михальченко, В.А. Русскин, С.М. Семенов, И.П. Орлянский, H. Sandor // Известия Томского политехнического университета. Инжиниринг георесурсов. - 2018. - Т. 329. № 3. - C. 102-112.

23. Obukhov S.G., Plotnikov I.A., Masolov V.G. Mathematical model of solar radiation based on climatological data from NASA SSE // IOP Conference Series: Materials Science and Engineering. - 2018. - V. 363 (012021). - 7 p. DOI: 10.1088/1757-899X/363/1/012021.

24. Обухов С.Г., Дураев Н.Н., Плотников И.А. Имитационная модель дизельного двигателя для исследования его рабочих характеристик на переменной частоте вращения // Известия Томского политехнического университета. - 2013. - Т. 322. № 4. - C. $48-52$.

25. Обухов С.Г., Сарсикеев Е.Ж. Математическая модель ветротурбины малой мощности в MATLAB SIMULINK // Альтернативная энергетика и экология: Международный научный журнал. - 2012. - № 2. - С. 42-48.

26. Mathematical modeling of hybrid renewable energy system: a review on small hydro-solar-wind power generation / B. Bhandari, S.R. Poudel, K.T. Lee, S.H. Ahn // International Journal of precision engineering and manufacturing-green technology. - 2014. - V. 1. - № 2. - P. 157-173.

27. A hybrid renewable system based on wind and solar energy coupled with an electrical storage: dynamic simulation and economic assessment / A. Buonomano, F. Calise, M.D. d'Accadia M. Vicidomini // Energy. - 2018. - V. 155. - P. 174-189.

28. Обухов С.Г., Плотников И.А. Имитационная модель режимов работы автономной фотоэлектрической станции с учетом реальных условий эксплуатации // Известия Томского политехнического университета. Инжиниринг георесурсов. 2017. - T. 328. - № 6. - C. 38-51.

29. Клевцов А.Л. Программа для расчета надежности информационных и управляющих систем АЭС // Ядерна та радіаційна безпека. - 2013. - № 1. - Р. 48-53.

30. Яковлева Э.В., Бабурин С.В. Расчет надежности электротехнического комплекса с фотоэлектрической станцией // Coвременная техника и технологии. - 2015. - № 5. - URL: http://technology.snauka.ru/2015/05/7197 (дата обращения: 15.07.2019)

31. Обухов С.Г., Плотников И.А., Масолов В.Г. Анализ режимов работы накопителей энергии в автономных гибридных электростанциях с возобновляемыми источниками энергии // Альтернативная энергетика и экология: Международный научный журнал. - 2018. - № 13-15. - С. 55-67.

Поступила 12.09.2019 г.

\section{Информация об авторах}

Обухов С.Г., доктор технических наук, профессор отделения электроэнергетики и электротехники Инженерной школы энергетики Национального исследовательского Томского политехнического университета.

Плотников И.А., кандидат технических наук, доцент отделения электроэнергетики и электротехники Инженерной школы энергетики Национального исследовательского Томского политехнического университета.

Ибрагим А., аспирант Инженерной школы энергетики Национального исследовательского Томского политехнического университета.

Масолов В.Г., кандидат технических наук, генеральный директор ООО «ВДМ-техника». 
UDC 621.311.26: 621.311 .68

\title{
DUAL ENERGY STORAGE FOR HYBRID ENERGY SYSTEMS WITH RENEWABLE ENERGY SOURCES
}

\author{
Sergey G. Obukhov ${ }^{1}$, \\ serob99@mail.ru \\ Igor A. Plotnikov ${ }^{1}$, \\ igorplt@tpu.ru \\ Ahmed Ibrahim ${ }^{1}$, \\ ibragim@tpu.ru \\ Vladimir G. Masolov², \\ vdm-tech@mail.ru \\ 1 National Research Tomsk Polytechnic University, \\ 30, Lenin avenue, Tomsk, 634050, Russia. \\ 2 VDM-tekhnika LLC, \\ 27, Pavlovskaya street, Moscow, 115093, Russia.
}

Relevance. The urgent task of technical and economic development of the northern and eastern regions of Russia is to provide reliable and efficient power supply to consumers, geographically located in remote, hard-to-reach areas. The use of hybrid energy systems with renewable energy sources is a promising way to solve this problem. A characteristic feature of the modes of hybrid systems, especially with a high level of fuel replacement, is the presence of ripples in charge-discharge currents of batteries used as energy storage devices. The operation of batteries in the mode of pulsed currents leads to rapid degradation of their characteristics and reduction in the service life, which leads to decrease in reliability of the power supply system and increase in the cost of generated electricity. A significant drawback of hybrid systems built according to well-known standard schemes is the inefficient use of the potential of primary renewable energy, which is especially critical for power systems geographically located in areas with severe climatic conditions. The article proposes technical solutions to eliminate the indicated problems.

Aim of the study is to search for and develop technical solutions to improve the efficiency of hybrid energy systems with a high level of fuel substitution.

Methods: mathematical and computer modeling using the Matlab/Simulink software environment.

Results. We offer a new method of construction and control algorithm of the modes of hybrid energy systems, which provide increasing their reliability and energy efficiency. The results of the modeling and the operating modes of a hybrid power plant with a high level of fuel substitution are presented, which proves that the proposed method of construction and the control algorithm provides reliable and efficient control of the power balance of a hybrid power system in all possible operating conditions. The application of the proposed algorithm in a dual-circuit energy storage device provides $5,7 \%$ reduction in the failure rate of the hybrid power system, increasing the operating life of the batteries by $50 \%$, more efficient use of renewable energy by $28 \%$ compared with conventional methods of construction of hybrid plants.

Key words:

Hybrid power system, renewable energy, energy storage, super capacitor, battery.

The work was supported by the Ministry of Science and Higher Education of the Russian Federation. The unique ID of the work - RFMEFI57617X0098 (Agreement on granting subsidies from 26.09.2017, no. 075-11-2018-181, internal agreement number 14.576.21.0098).

\section{REFERENCES}

1. Renewables 2018 Global Status Rep ort. Renewable Energy Policy Network for the 21 $1^{\text {st }}$ Century. 2018. 325 p. Available at: http://www.ren21.net/ (accessed 29 April 2019).

2. Sawle Y., Gupta S.C., Bohre A.K. Review of hybrid renewable energy systems with comparative analysis of off-grid hybrid system. Renewable and Sustainable Energy Reviews, 2018, vol. 81, no. 2, pp. 2217-2235.

3. Arkhipova O.V., Kovalev V.Z., Khamitov R.N. Methodology of modeling regionally isolated electrotechnical complex. Bulletin of the Tomsk Polytechnic University. Geo Assets Engineering, 2019, vol. 330, no. 1, pp. 173-180. In Rus.

4. Salas V., Suponthana W., Salas R.A. Overview of the off-grid photovoltaic diesel batteries systems with AC loads. Applied Energy, 2015, vol. 157, no. 1, pp. 195-216.
5. Battery storage for renewables: market status and technology outlook. International Renewable Energy Agency. Available at: https://www.irena.org/-

/media/Files/IRENA/Agency/Publication/2015/IRENA_Battery_ Storage_report_2015.pdf (accessed 29 April 2019).

6. Eller A., Gauntlett D. Energy storage trends and opportunities in emerging markets. Available at: https://www.esmap.org/sites/ default/files/esmap-files/7151-IFC-EnergyStorage-report.pdf (accessed 29 April 2019)

7. Hu X., Martinez C.M., Yang Y. Charging, power management, and battery degradation mitigation in plug-in hybrid electric vehicles: a unified cost-optimal approach. Mechanical Systems and Signal Processing, 2017, vol. 87, no. 8, pp. 4-16.

8. Kan S.Y., Verwaal M., Broekhuizen H. The use of batterycapacitor combinations in photovoltaic powered products. Journal of Power Sources, 2006, vol. 162, no. 2, pp. 971-974.

9. Lahyania A., Sari A., Lahbib I., Venet P. Optimal hybridization and amortized cost study of 
battery/supercapacitors system under pulsed loads. Journal of Energy Storage, 2016, vol. 6, pp. 222-231.

10. Hund T. Capacity loss in PV batteries and recovery procedures. Photovoltaic System Applications Department, Sandia National Laboratories. 12 p. Available at: https://pdfs.semanticscholar. org/87fd/d2343a6665f1251ee0c645fd3442f2e340e1.pdf?_ga=2.9 $1045971.85236334 .1578964892-1940863712.1578964892$ (accessed 29 April 2019).

11. Jossen A. Garche J., Sauer D.U. Operating conditions of batteries in PV applications. Solar Energy, 2004, vol. 76, pp. 759-769.

12. Jakhrani A.Q., Othman A-K., Rigit A.R.H., Samo S.R. Life cycle cost analysis of a standalone PV system. IEEE 2012 Int. Conf. Green Ubiquitous Technol. Jakarta, Indonesia, 2012. pp. 82-85.

13. Ataei A., Nedaei M., Rashidi R., Yoo C. Optimum design of an off-grid hybrid renewable energy system for an office building. Journal of Renewable and Sustainable Energy, 2015, vol. 7 (053123). 27 p. DOI: 10.1063/1.4934659

14. Nehrir M.H., Wang C., Strunz K., Aki H., Ramakumar R., Bing J., Miao Z., Salameh Z. A review of hybrid renewable/alternative energy systems for electric power generation: configurations, control, and applications. IEEE Transactions on Sustainable Energy, 2011, vol. 2, no. 4, pp. 392-403.

15. Upadhyay S., Sharma M.P. A review on configurations, control and sizing methodologies of hybrid energy systems. Renewable and Sustainable Energy Reviews, 2014, vol. 38, pp. 47-63.

16. Sawle Y., Gupta S.C., Bohre A.K. PV-wind hybrid system: a review with case study. Cogent Engineering, 2016, vol. 3, pp. 1-21.

17. Obuhov S.G., Plotnikov I.A. Comparative analysis of the schemes of autonomous power plants using renewable energy installations. Promyshlennaya energetika, 2012, no. 7, pp. 46-51. In Rus.

18. Olatomiwa L., Mekhilef S., Ismail M.S., Moghavvemi M. Energy management strategies in hybrid renewable energy systems: a review. Renewable and Sustainable Energy Review, 2016, vol. 62, pp. 821-835.

19. Chauhan A., Saini R.P. A review on integrated renewable energy system based power generation for stand-alone applications: configurations, storage options, sizing methodologies and control. Renewable and Sustainable Energy Reviews, 2014, vol. 38, pp. 99-120.

20. Grigoriev A.S., Skorlygin V.V., Grigoriev S.A., Melnik D.A., Filimonov M.N. A hybrid power plant based on renewables and electrochemical energy storage and generation systems for decentralized electricity supply of the northern territories. International Journal of Electrochemical Science, 2018, vol. 13, pp. $1822-1830$.

21. Obukhov S.G., Plotnikov I.A., Popov M.M., Surkov M.A. Sistema avtonomnogo elektrosnabzheniya [Self-contained electric power supply system]. Patent RF, no. 2638025, 2017.
22. Mikhalchenko S.G., Russkin V.A., Semenov S.M., Orlyanskiy I.P., Halasz S. Construction of adaptive algorithm of power extreme control in solar energy system. Bulletin of the Tomsk Polytechnic University. Geo Assets Engineering, 2018, vol. 329, no. 3, pp. 102-112. In Rus.

23. Obukhov S.G., Plotnikov I.A., Masolov V.G. Mathematical model of solar radiation based on climatological data from NASA SSE. IOP Conference Series: Materials Science and Engineering. 2018, vol. 363 (012021), 7 p. DOI: 10.1088/1757899X/363/1/012021

24. Obukhov S.G., Duraev N.N., Plotnikov I.A. Simulation model of the diesel engine to investigate its performance on variable speed. Bulletin of the Tomsk Polytechnic University, 2013, vol. 322, no. 4, pp. 48-52. In Rus.

25. Obukhov S.G., Sarsikeyev E.Zh. The mathematical model of small-scale wind turbine using MATLAB Simulink software. International Scientific Journal for Alternative Energy and Ecology, 2012, no. 2, pp. 42-48. In Rus.

26. Bhandari B., Poudel S.R., Lee K.T., Ahn S. H. Mathematical modeling of hybrid renewable energy system: a review on small hydro-solar-wind power generation. International Journal of precision engineering and manufacturing-green technology, 2014, vol. 1, no. 2, pp. 157-173.

27. Buonomano A., Calise F., D'Accadia M.D., Vicidomini M. A hybrid renewable system based on wind and solar energy coupled with an electrical storage: dynamic simulation and economic assessment. Energy, 2018, vol. 155, pp. 174-189.

28. Obukhov S.G., Plotnikov I.A. Simulation model of operation of autonomus photovoltaic plant under actual operating condition. Bulletin of the Tomsk Polytechnic University. Geo Assets Engineering, 2017, vol. 328, no. 6, pp. 38-51. In Rus.

29. Klevtsov A.L. Software for calculation of reliability of NPP instrumentation and control systems. Yadernaya $i$ radiatsionnaya bezopasnost, 2013, no. 1, pp. 48-53. In Rus.

30. Yakovleva E.V., Baburin S.V. Calculation of reliability of the electrical complex with a photovoltaic station. Sovremennaya tekhnika $i$ tekhnologii, 2015, no. 5. In Rus. Available at: http://technology.snauka.ru/2015/05/7197 (accessed 15 July 2019).

31. Obukhov S.G., Plotnikov I.A., Masolov V.G. The analysis of operation modes of energy stores in autonomous hybrid power plants with renewable energy resources. International Scientific Journal for Alternative Energy and Ecology, 2018, no. 13-15, pp. 55-67. In Rus.

\section{Information about the authors}

Sergey G. Obukhov, Dr. Sc., professor, National Research Tomsk Polytechnic University.

Igor A. Plotnikov, Cand. Sc., associate professor, National Research Tomsk Polytechnic University.

Ahmed Ibrahim, postgraduate student, National Research Tomsk Polytechnic University.

Vladimir G. Masolov, Cand. Sc., general manager, VDM-tekhnika LLC. 